Research Article

\title{
Solution of the Falkner-Skan Equation Using the Chebyshev Series in Matrix Form
}

\author{
Abdelrady Okasha Elnady ${ }^{(D},{ }^{1}$ M. Fayek Abd Rabbo, ${ }^{2}$ and Hani M. Negm ${ }^{3}$ \\ ${ }^{1}$ Mechatronics Department, Faculty of Engineering, O6University, Cairo, Egypt \\ ${ }^{2}$ Mechanical Engineering Department, Shoubra Faculty of Engineering, Benha University, Banha, Egypt \\ ${ }^{3}$ Aerospace Engineering Department, Cairo University, Giza, Egypt \\ Correspondence should be addressed to Abdelrady Okasha Elnady; rady_nady@yahoo.com
}

Received 2 November 2019; Revised 10 February 2020; Accepted 26 February 2020; Published 1 May 2020

Academic Editor: Xing Ma

Copyright (C) 2020 Abdelrady Okasha Elnady et al. This is an open access article distributed under the Creative Commons Attribution License, which permits unrestricted use, distribution, and reproduction in any medium, provided the original work is properly cited.

\begin{abstract}
A numerical method for the solution of the Falkner-Skan equation, which is a nonlinear differential equation, is presented. The method has been derived by truncating the semi-infinite domain of the problem to a finite domain and then expanding the required approximate solution as the elements of the Chebyshev series. Using matrix representation of a function and their derivatives, the problem is reduced to a system of algebraic equations in a simple way. From the computational point of view, the results are in excellent agreement with those presented in published works.
\end{abstract}

\section{Introduction}

Ordinary differential equations are important tools in solving real-world problems. Several natural phenomena are modelled by ODEs that have been used in several fields, such as physics, engineering, and biology [1-7].

The Falkner-Skan equation arises in the study of laminar boundary layers exhibiting similarity. The similarity solutions of the two-dimensional incompressible laminar boundary layer equations are well-known as the Falkner-Skan equation. The F-S equation is a one-dimensional third-order nonlinear two-point boundaryvalue problem which has no closed-form solution. The problem is given by

$$
f^{\prime \prime \prime}+\beta_{0} f f^{\prime \prime}+\beta\left(1-f^{\prime 2}\right)=0, \quad 0 \leq \eta<\infty,
$$

where $\beta_{0}$ and $\beta$ are constants and primes denote differentiation with respect to $\eta$. The associated boundary conditions are given by

$$
\begin{aligned}
f(0) & =0, \\
f^{\prime}(0) & =0, \\
\lim _{\eta \rightarrow \infty} f^{\prime}(\eta) & =1 .
\end{aligned}
$$

The solution of (1) and (2) is characterized by $f^{\prime \prime}(0)=\alpha$. The numerical treatment of this problem was addressed by many authors, namely, Lakestani [8], Parand et al. [9], ElNady and Abd Rabbo [10, 11], Cebeci and Keller [12], Na [13], Asaithambi [14], Asaithambi [15], Elgazery [16], and Ganapol [17]. These techniques have mainly used shooting algorithms or invariant imbedding. The Chebshev collocation matrix method [18] has been presented the numerical solution of nonlinear differential equations. The method in [18] transforms the nonlinear differential equation into the matrix equation, which corresponds to a system of nonlinear algebraic equations with unknown Chebyshev coefficients, via Chebyshev collocation points. The method in [19] transforms the nonlinear differential equation into the system of nonlinear algebraic equations with unknown 
shifted Chebyshev coefficients, via Chebyshev-Gauss collocation points. The solution of this system yields the Chebyshev coefficients of the solution function. The method is valid for both initial-value and boundary-value problems.

The purpose of this paper is to develop an efficient method based on the Chebyshev series which is much more straightforward and simpler than the other existing algorithms. Using matrix notation of the Chebyshev series, the nonlinear differential equation converts into a system of algebraic equations which can be easily solved. The importance of the present method arises from its simplicity and the fact that it does not require to guess the value of $f^{\prime \prime}(0)$.

\section{Chebyshev Series}

Any continuous function $f(\xi)$ in the interval $0 \leq \xi \leq 1$ can be written in a Chebyshev series as follows [20]:

$$
f(\xi)=\sum_{r=0}^{\infty}{ }^{+} a T_{r}(\xi)
$$

The series expansion (3) is fast-converging, and a good approximation is obtained by taking a few terms. Therefore, equation (3) is approximated by

$$
f(\xi)=\sum_{r=0}^{N}{ }^{+} a_{r} T_{r}(\xi)
$$

where + sign means that the $1^{\text {st }}$ term must be halved and $a_{r}$ are constants to be determined so as to obtain the best possible fit.

$$
\begin{aligned}
T_{r}(\xi) & =\operatorname{Cos}(r \quad t), \\
\operatorname{Cos}(t) & =2 \xi-1, \quad 0 \leq \xi \leq 1 .
\end{aligned}
$$

The shifted Chebyshev polynomials satisfy the recurrence relations

$$
\begin{aligned}
T_{r+1}(\xi) & =2(2 \xi-1) T_{r}(\xi) \operatorname{Tr}-1(\xi), \quad 2 \leq r \leq N, \\
T_{0} & =1 \\
T_{1} & =2 \xi-1,
\end{aligned}
$$

and the orthogonality conditions

$$
\int_{0}^{1} \frac{\left(T_{m}(\xi) T_{n}(\xi)\right)}{(\sqrt{\xi} \sqrt{1-\xi})} d \xi= \begin{cases}0, & \text { for } m \neq n \\ \frac{\pi}{2}, & \text { for } m=n \neq 0 \\ \pi, & \text { for } m=n=0\end{cases}
$$

where for a known function $f(\xi)$, the coefficients are given by

$$
a_{r}=\frac{2}{\pi} \int_{0}^{1} \frac{f(\xi) T_{r}(\xi)}{\sqrt{\xi} \sqrt{1-\xi}} \mathrm{d} \xi, \quad 0 \leq r \leq N .
$$

Also, the derivatives of the function $f(\xi)$ can be expanded in a Chebyshev series keeping a recurrence relation between the coefficients of the function and its derivatives. The first derivative $f^{\prime}(\xi)$ is expressed in a Chebyshev series as [20]

$$
f^{\prime}(\xi)=\sum_{r=0}^{N-1}{ }^{+} a_{r}^{(1)} T_{r}(\xi) .
$$

The coefficients $a_{r}^{(1)}$ satisfy the recursive relation

$$
a_{r-1}^{(1)}-a_{r+1}^{(1)}=4 r a_{r}, \quad 1 \leq r \leq N .
$$

Similarly, the $m^{\text {th }}$ derivative is written as

$$
f^{m}(\xi)=\sum_{r=0}^{N-m}{ }^{+} a_{r}^{(m)} T_{r}(\xi)
$$

where $a_{r-1}^{(m)}-a_{r+1}^{(m)}=4 r a_{r}^{(m-1)}, 1 \leq r \leq N-(m-1)$.

2.1. Matrix Representation of Function and Function Derivatives. Any continuous function $f(\xi)$ in the interval $0 \leq \xi \leq 1$ and its derivatives can be written in a matrix form of the Chebyshev series as follows:

$$
f(\xi)=\left[T_{r}\right][I]\left\{a_{i}\right\}, \quad r=0,1,2, \ldots, N, i=0,1,2, \ldots, N .
$$

The first-order-derivative coefficients $\left\{a_{r}^{(1)}\right\}$ in equation (10) can be written in terms of the original function coefficients $\left\{a_{i}\right\}$ using matrix notation as follows:

$$
\left\{a_{r}^{(1)}\right\}=4[A]\left\{a_{i}\right\}, \quad r=0,1,2, \ldots, N-1, i=1,2,3, \ldots, N,
$$

where $[A]$ is an upper triangular matrix of order $N \times N$.

The elements of the matrix $a_{i j}$ are defined as

$$
a_{i j}= \begin{cases}0, & i>j \text { or } i+j \text { odd }, \\ j, & i \leq j \text { and } i+j \text { even. }\end{cases}
$$

The form of matrix $[A]$ for $N=5$ for example is

$$
[A]=\left[\begin{array}{lllll}
1 & 0 & 3 & 0 & 5 \\
0 & 2 & 0 & 4 & 0 \\
0 & 0 & 3 & 0 & 5 \\
0 & 0 & 0 & 4 & 0 \\
0 & 0 & 0 & 0 & 5
\end{array}\right] .
$$

From equation (13), it is noted that the first-order-derivative coefficients are written in terms of the $N$ coefficients $\left\{a_{i}\right\},(i=1,2, \ldots, N)$ of the function $f(\xi)$. To represent $\left\{a_{r}^{(1)}\right\}$ in terms of all function coefficients $\left\{a_{i}\right\}, i=0,1,2, \ldots, N$, we add a new left column with zero entries in the matrix $[A]$, and the new matrix is termed [A01]. Thus,

$$
\begin{aligned}
\left\{a_{r}^{(1)}\right\}= & 4[A 01]\left\{a_{i}\right\}, \\
& \quad r=0,1,2, \ldots, N-1, i=1,2,3, \ldots, N,
\end{aligned}
$$

where [A01] is of order $N \times N+1$ and takes; for $N=5$, the form 


$$
[A 01]=\left[\begin{array}{llllll}
0 & 1 & 0 & 3 & 0 & 5 \\
0 & 0 & 2 & 0 & 4 & 0 \\
0 & 0 & 0 & 3 & 0 & 5 \\
0 & 0 & 0 & 0 & 4 & 0 \\
0 & 0 & 0 & 0 & 0 & 5
\end{array}\right] .
$$

The second-order-derivative coefficients $\left\{a_{r}^{(2)}\right\}$ can be written in terms of the function coefficients $\left\{a_{i}\right\}$ using matrix notation as follows:

$$
\begin{aligned}
& \left\{a_{r}^{(2)}\right\}=16[A]_{-1,-1}[A 01]\left\{a_{i}\right\}, \quad r=0,1,2, \ldots, N-2, \\
& \left\{a_{r}^{(2)}\right\}=16[A 02]\left\{a_{i}\right\}, \quad i=0,1,2, \ldots, N
\end{aligned}
$$

where [A02] is of order $N-1 \times N+1 .[A]_{-1,-1}$ is the matrix $[A]$ after deleting the last row and last column. ${ }^{-1}[A 01]$ is the matrix $[A 01]$ after deleting the first row.

$$
[A 02]=\left[\begin{array}{llll}
1 & 0 & 3 & 0 \\
0 & 2 & 0 & 4 \\
0 & 0 & 3 & 0 \\
0 & 0 & 0 & 4
\end{array}\right] *\left[\begin{array}{llllll}
0 & 0 & 2 & 0 & 4 & 0 \\
0 & 0 & 0 & 3 & 0 & 5 \\
0 & 0 & 0 & 0 & 4 & 0 \\
0 & 0 & 0 & 0 & 0 & 5
\end{array}\right]=\left[\begin{array}{cccccc}
0 & 0 & 2 & 0 & 16 & 0 \\
0 & 0 & 0 & 6 & 0 & 30 \\
0 & 0 & 0 & 0 & 12 & 0 \\
0 & 0 & 0 & 0 & 0 & 20
\end{array}\right] \text {. }
$$

The general form of the $m^{\text {th }}$ derivative coefficients $\left\{a_{r}^{(m)}\right\}$ can be written in terms of the function coefficients $\left\{a_{i}\right\}$ using matrix notation as follows:

$$
\begin{gathered}
\left\{a_{r}^{(m)}\right\}=(4)^{m}[A]_{1-m, 1-m}{ }^{-1}[A 0(m-1)]\left\{a_{i}\right\}, \\
r=0,1,2, \ldots, N-m, \\
\left\{a_{r}^{(m)}\right\}=(4)^{m}[A 0 m]\left\{a_{i}\right\} ; \quad i=0,1,2, \ldots, N,
\end{gathered}
$$

where $m$ the order of derivative, $[A]_{1-m, 1-m}$ matrix $[A]$ after deleting the last $(m-1)$ rows and $(m-1)$ columns. ${ }^{-1}[]$ matrix [ ] after deleting the first row.

$$
\begin{aligned}
f^{m}(\xi)=(4)^{m}\left[T_{r}\right][A 0 m]\left\{a_{i}\right\}, \\
r=0,1,2, \ldots, N-m, i=0,1,2, \ldots, N,
\end{aligned}
$$

where $\left[T_{r}\right]$ is a row matrix whose elements are $T_{r}(\xi)$. Note that in the matrix form the first term of $\left[T_{r}\right]$ must be halved.

Now, consider the general nonhomogenous differential equation of $\mathrm{m}^{\text {th }}$ order.

$$
f^{m}+f^{m-1}+f^{m-2}+\cdots+f^{\prime}+f=P .
$$

After expanding each term in the Chebyshev series, the abovementioned differential equation can be written as

$$
\begin{aligned}
& \sum_{r=0}^{N-m}{ }^{+} a_{r}^{(m)} T_{r}(\xi)+\sum_{r=0}^{N-(m-1)}{ }^{+} a_{r}^{(m-1)} T_{r}(\xi)+\cdots+\sum_{r=0}^{N-1}{ }^{+} a_{r}^{(1)} T_{r}(\xi) \\
& \quad+\sum_{r=0}^{N}{ }^{+} a_{r} T_{r}(\xi)=\sum_{r=0}^{N}{ }^{+} p_{r} T_{r}(\xi) .
\end{aligned}
$$

The forcing-function coefficients $p_{r}$ can be evaluated using equation (8). Equating the coefficients of like Chebyshev polynomial terms on either side, the resulting $N+1-m$ algebraic equations can be written in a matrix form using equations (16) and (21) as

$$
\begin{aligned}
& {\left[4^{(m)}[A 0 m]+4^{(m-1)}[A 0(m-1)]+\cdots+4[A 01]+[I]\right]} \\
& \quad\left\{a_{i}\right\}=\left\{p_{r}\right\} ; \quad r=0,1,2, \ldots, N+1-m, i=0,1,2, \ldots, N,
\end{aligned}
$$

where all matrices in equation (24) are of the same order $(N+1-m \times N+1)$. For all derivatives lower than the highest derivative, the first $N+1-m$ rows are chosen so as to satisfy equation (24). In order to be able to solve equation (24), $m$ additional equations are needed. These additional equations are supplied by the problem boundary conditions.

2.2. Matrix Representation of Function Products. If $f(\xi)$ and $g(\xi)$ are two continuous functions represented by truncated Chebyshev series as

$$
\begin{aligned}
& f(\xi)=\sum_{r=0}^{N}{ }^{+} a_{r} T_{r}(\xi), \\
& g(\xi)=\sum_{r=0}^{M}{ }^{+} b_{r} T_{r}(\xi),
\end{aligned}
$$

then the product of these functions can be written in a Chebyshev series as

$$
g(\xi) f(\xi)=\sum_{r=0}^{M+N}{ }^{+} c_{r} T_{r}(\xi),
$$

where

$$
\begin{aligned}
& c_{o}= \sum_{i=0}^{N+M}+{ }^{+} a_{i} b_{i}, \\
& c_{r}=\frac{1}{2} \sum_{i=0}^{N+M}+{ }_{i}\left(b_{i+r}+b_{|i-r|}\right), \\
& \quad 1 \leq r \leq M+N, i+r \leq M,|i-r| \leq M \forall i .
\end{aligned}
$$

The $\left\{c_{r}\right\}$ coefficients can be written in terms of the $\left\{a_{i}\right\}$ coefficients only using matrix notation as follows:

$$
\left\{c_{r}\right\}=[H]\left\{a_{i}\right\},
$$

where $r=0,1,2, \ldots, N+M, i=0,1,2, \ldots, N,\left\{c_{r}\right\}$ is a column matrix of order $N+M+1 \times 1,[H]$ is rectangular matrix of 
order $N+M+1 \times N+1$, and $\left\{a_{i}\right\}$ is a column matrix of order $N+1 \times 1$.

The elements $h_{i j}$ of the matrix $[H]$ can be written as

$$
h_{i j}=\left\{\begin{array}{cc}
\frac{1}{4}\left(b_{|i-j|}+b_{i+j-2}\right), & \text { for } i=1,2, \ldots, N+M+1, j=1, \\
\frac{1}{2}\left(b_{|i-j|}+b_{i+j-2}\right), & \text { for } i=1,2, \ldots, N+M+1, \\
& j=2,3, \ldots, N+1, \\
|i-j| \leq M, i+j-2 \leq M, \forall i, j .
\end{array}\right.
$$

Therefore, equation (28) takes the form

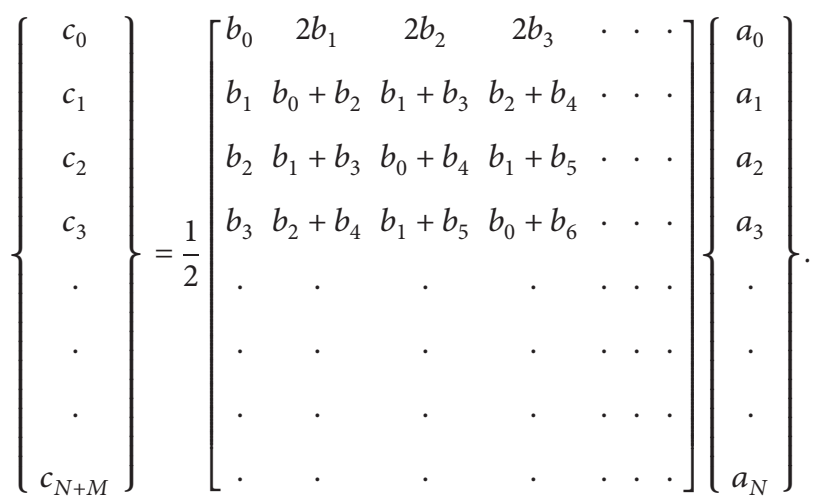

\section{Application to the Falkner-Skan Equation}

The purpose of the present section is to solve the Falkner-Skan equations (1) and (2) numerically by using the Chebyshev series in a matrix form. Again, to solve this problem, the semi-infinite physical domain $[0, \infty]$ of the problem is truncated to a finite domain $[0, L]$, where $L$ is a sufficiently large number. The Falkner-Skan equation (1) can be written in the nondimensional form as

$$
\frac{1}{L^{3}} \frac{\mathrm{d}^{3} f(\xi)}{\mathrm{d} \xi^{3}}+\beta_{0} f(\xi) \frac{1}{L^{2}} \frac{\mathrm{d}^{2} f(\xi)}{\mathrm{d} \xi^{2}}-\beta \frac{1}{L} \frac{\mathrm{d} f(\xi)}{\mathrm{d} \xi} \frac{1}{L} \frac{\mathrm{d} f(\xi)}{\mathrm{d} \xi}=\beta, \quad \xi=\frac{\eta}{L} .
$$

The associated boundary conditions (2) are written in the nondimensional form as

$$
\begin{array}{r}
f(0)=0 \\
\frac{1}{L} \frac{\mathrm{d} f(0)}{\mathrm{d} \xi}=0 \\
\frac{1}{L} \frac{\mathrm{d} f(1)}{\mathrm{d} \xi}=1
\end{array}
$$

Expanding $f(\xi)$ in $(N+1)$-Chebyshev terms, we get $N+1$ unknown coefficients. Now, using matrix notation for the functions, function derivatives, and functions products and applying the rule of matrix multiplication, equation (31) can be written as a system of algebraic equations in the following matrix form:

$$
\left[\frac{64}{L^{3}}[A 03]+\beta_{0} \frac{16}{L^{2}}[H b]-\beta \frac{4}{L}[H a] \frac{4}{L}[A 01]\right]\left\{f_{i}\right\}=\left\{\beta_{r}\right\},
$$

where $[\mathrm{Ha}]$ is the matrix of coefficients of the first derivative, $[\mathrm{Hb}]$ is the matrix of coefficients of the second derivative, $\left\{f_{i}\right\}$ are Chebyshev coefficients of the function $f$, and $\left\{\beta_{r}\right\}$ are Chebyshev coefficients of $\beta$ and obtained from equation (8).

The highest derivative in equation (33) is of order 3 , so the number of algebraic equations is $N-2$ along with 3 boundary conditions at $\xi=0$ and $\xi=1$, leading to $N+1$ equations in $N+1$ unknowns, which can be easily solved. It is to be noted that all matrices in equation (33) are of order $(N-2 \times N+1)$.

3.1. Boundary Conditions. Fortunately, it is relatively easy to represent any boundary conditions for the functions expanded in the Chebyshev series. The boundary conditions given in (32) can be written as

$$
\begin{array}{r}
{[T R 0]\left\{f_{i}\right\}=0,} \\
\frac{4}{L}[T R 01][A 01]\left\{f_{i}\right\}=\frac{4}{L}[T 0 A 01]\left\{f_{i}\right\}=0, \\
\frac{4}{L}[T R 11][A 01]\left\{f_{i}\right\}=\frac{4}{L}[T 1 A 01]\left\{f_{i}\right\}=1,
\end{array}
$$

where [TR0] is a row matrix of $N+1$ Chebeychev terms at $\xi=0,[T R 01]$ is a row matrix of $N$ Chebeychev terms at $\xi=0$, and [TR11] is a row matrix of $N$ Chebeychev terms at $\xi=1$. Note: the first term of Chebyshev terms must be halved.

3.2. Procedure of Solution. The present algorithm consists of the following procedure:

(a) It is started with a relatively small value of $L$ as the initial value for semi-infinite domain.

(b) The system of equations (33) and (34) are solved simultaneously to find the velocity profile $f^{\prime}(\eta)$.

(c) $L$ is continuously increased up to $\lim _{\eta \rightarrow \infty} f^{\prime}(\eta)=$ 1. This is achieved if two successive values $f^{\prime \prime}(0)$ differ by about $1 E-6$. Figure 1 shows the results for $\beta_{0}=1$ and $\beta=2$.

\section{Results and Discussion}

The Falkner-Skan equation has two coefficients $\beta_{0}$ and $\beta$. The solutions corresponding to $\beta=0$ have been known as constant flow, those corresponding to $\beta>0$ are called accelerating flow, and those corresponding to $\beta<0$ are known as decelerating flow. Physically relevant solutions exist only for $-0.1988<\beta \leq 2$. If $\beta_{0}=1 / 2$ and $\beta=0$, it is called Blasius flow; if $\beta_{0}=1$ and $\beta=1 / 2$, it describes the Homann axisymmetric stagnation flow, if $\beta_{0}=1$ and $\beta=1$, 


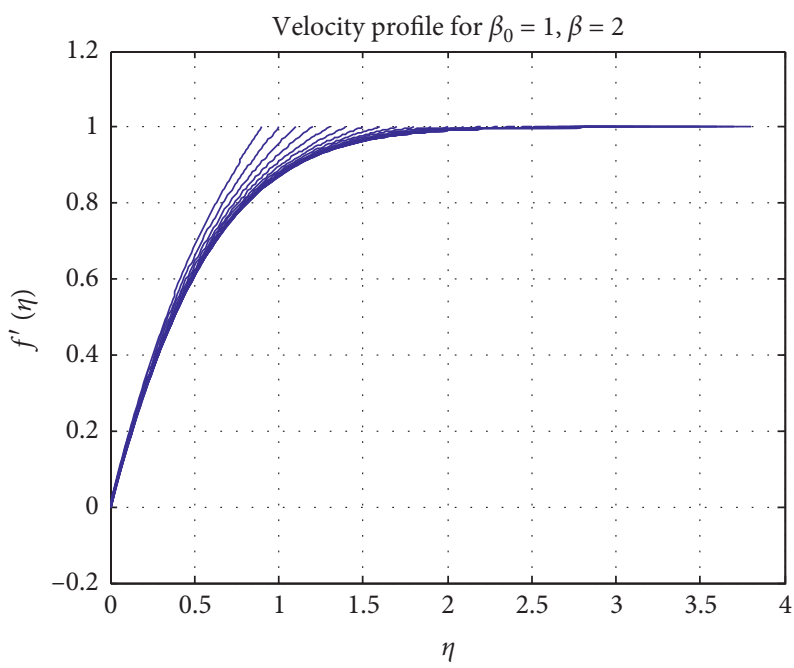

Figure 1: Velocity profile for $\left(\beta_{0}=1\right.$ and $\left.\beta=2\right)$.

it describes the Hiemenz flow, if $\beta_{0}=0$ and $\beta=1$, it is called the Pohlhausen flow, and if $\beta_{0}=2$ and $\beta=1$, it represents the problem of Homann, describing the steady flow in the boundary layer along a surface of revolution near the stagnation point. Sometimes, the Falkner-Skan equation specifically refers to $\beta_{0}=1$.

The Falkner-Skan equation was solved using the proposed Chebyshev series in a matrix form for different values of $\beta_{0}$ and $\beta$. It was noted that the solution is stable and convergent even for small number of terms less than ten. Finally, in all solutions, the number of Chebyshev terms is taken $N=20$. Table 1 compares the initial slope $f^{\prime \prime}(0)$ obtained using the present method with the values obtained in [17] by using highly accurate algorithm based on a Maclaurin series representation. It is seen that the present method delivers the same order of accuracy.

It is noted that $L$ has a great effect on the solution. The results show that the system equations (33) and (34) have a solution with high level of accuracy for different values of the semi-infinite interval $L$ as presented in Table 2 .

The velocity profile for different flows mentioned above is obtained using the present technique and Runge-Kutta $4^{\text {th }}$ order method and presented in the following figures.

Figure 2 shows the velocity profile of the Blasius flow for $\beta_{0}=1 / 2$ and $\beta=0$. Figure 3 shows the velocity profile of the Homann axisymmetric stagnation flow for $\beta_{0}=1$ and $\beta=1 / 2$. Figure 4 shows the velocity profile of the Hiemenz flow for $\beta_{0}=1$ and $\beta=1$. It is clear that the present solution has an excellent agreement with that obtained by the Runge-Kutta $4^{\text {th }}$ order method. The solutions shown are not a single solution, but it is a set of solutions for different lengths and gives the same value of $f^{\prime \prime}(0)$ as presented in Table 2.

Figure 5 shows the velocity profile of the Pohlhausen flow for $\beta_{0}=0$ and $\beta=1$. It is clear in this figure that the
TABLE 1: Comparison of $f^{\prime \prime}(0)$ for $\beta_{0}=1$ and different values of $\beta$.

\begin{tabular}{lcccc}
\hline$\beta$ & $f^{\prime \prime}(0)[17]$ & Present method & Absolute error & $L$ \\
\hline 40 & 7.314784974 & 7.314784949 & $2.5 E-08$ & 1.19 \\
30 & 6.338208628 & 6.338208608 & $2.0 E-08$ & 1.35 \\
20 & 5.180718025 & 5.180718025 & $3.9 E-10$ & 1.61 \\
15 & 4.491486898 & 4.491486992 & $-9.4 E-08$ & 1.78 \\
10 & 3.675234101 & 3.675234108 & $-6.8 E-09$ & 2.18 \\
2 & 1.687218169 & 1.687218169 & $3.1 E-11$ & 4.37 \\
1 & 1.232587657 & 1.232588439 & $-7.8 E-07$ & 4.30 \\
0.5 & 0.92768004 & 0.927686117 & $-6.1 E-06$ & 4.41 \\
0 & 0.469599988 & 0.4704412 & $-8.4 E-04$ & 4.03 \\
\hline
\end{tabular}

solution obtained by the Runge-Kutta $4^{\text {th }}$ order method is divergent at $L$ nearly equal 3.25 while the present solution is still convergent until $L=6.98476$ which means that the present solution is powerful. This note is valid for all values of $\beta$ and $\beta_{0}=0$.

Figure 6 shows the velocity profile of the problem of Homann describing steady flow in the boundary layer along a surface of revolution near the stagnation point in which $\beta_{0}=2$ and $\beta=1$. As mentioned previously, the present solution has an excellent agreement with that obtained by the Runge-Kutta $4^{\text {th }}$ order method.

The values of $f^{\prime \prime}(0)$ and the largest $L$ for each case are presented.

For $\beta_{0}=1$ and $\beta<0$, two families of solutions are obtained. One family of solutions corresponds to forward flow and the other corresponds to reverse flow as shown in Figures 7 and 8 .

The step of increasing $L$ is 0.05 , and the error between two consecutive values of $f^{\prime \prime}(0)$ is $1 E-2$.

Refining $\Delta L=0.001$ and adopting the error between two consecutive values of $f^{\prime \prime}(0)$ to be $1 E-6$, the results are shown in Figures 9 and 10. 
TABLE 2: Effect of $\eta$ on $f^{\prime \prime}(0)$ for $\beta=40$ and $\beta_{0}=1$ (error $\left.=1 E-7\right)$.

\begin{tabular}{lcccccc}
\hline$\eta$ & 1.01 & 1.02 & 1.03 & 1.04 & 1.05 & 1.06 \\
$f^{\prime \prime}(0)$ & 7.3147854 & 7.3147853 & 7.3147852 & 7.3147852 & 7.3147851 & 7.3147851 \\
$\eta$ & 1.07 & 1.08 & 1.09 & 1.1 & 1.11 & 7.12 \\
$f^{\prime \prime}(0)$ & 7.3147851 & 7.3147850 & 7.3147850 & 7.3147849 & 7.3147849 & 7.3147849 \\
$\eta$ & 1.13 & 1.14 & 1.15 & 1.16 & 7.19 & 7.3147857 \\
$f^{\prime \prime}(0)$ & 7.3147848 & 7.3147848 & 7.3147847 & 7.3147846 & \\
\hline
\end{tabular}

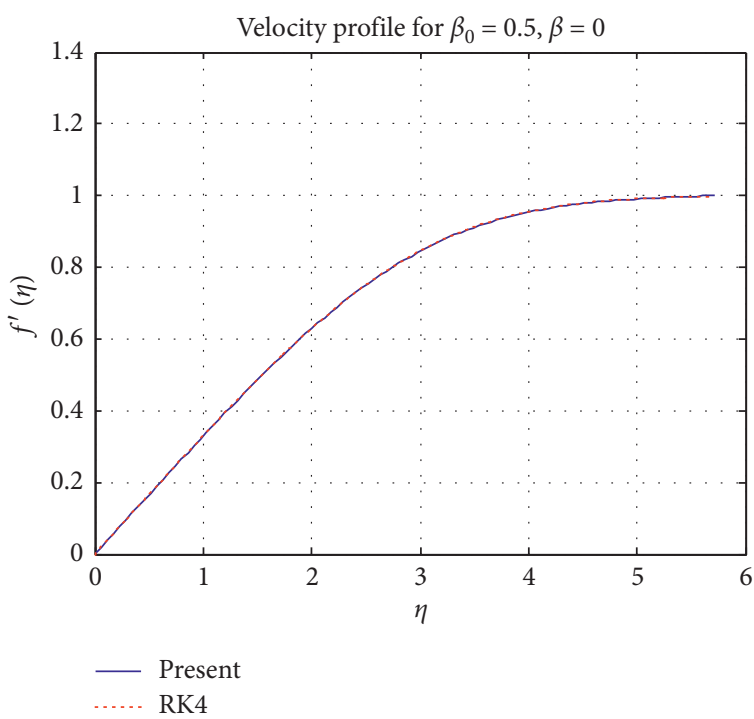

FIGURE 2: Velocity profile of the Blasius flow $\left(\beta_{0}=1 / 2\right.$ and $\left.\beta=0\right)$. $f^{\prime \prime}(0)=0.332057336151$ for $L=5.7205339053$.

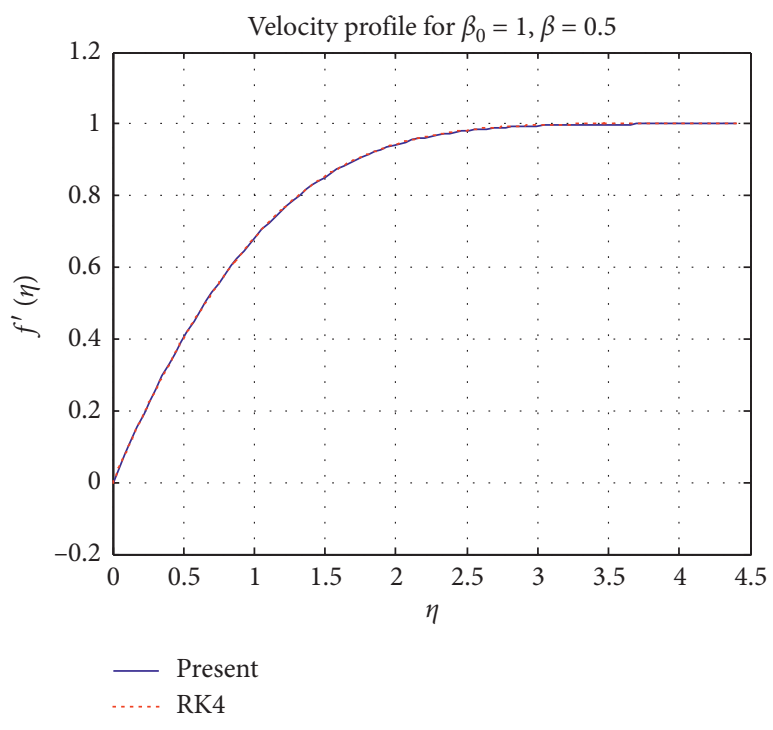

FIGURE 3: Velocity profile of the Homann axisymmetric stagnation flow $\left(\beta_{0}=1\right.$ and $\left.\beta=1 / 2\right)$. $f^{\prime \prime}(0)=0.927686124423$ for $L=4.404$.

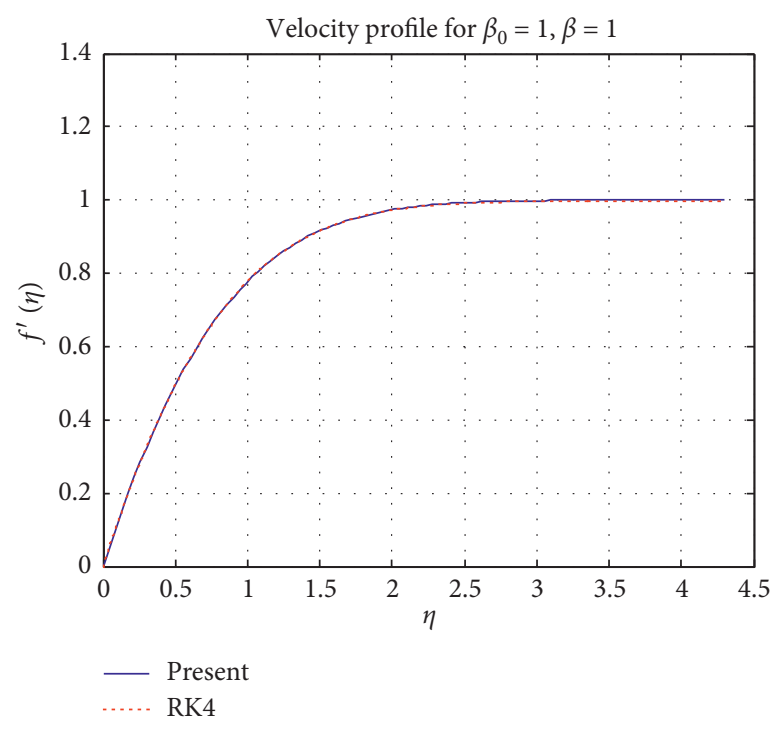

Figure 4: Velocity profile of the Hiemenz flow $\left(\beta_{0}=1\right.$ and $\left.\beta=1\right)$. $f^{\prime \prime}(0)=1.232588438646$ for $L=4.3$. 


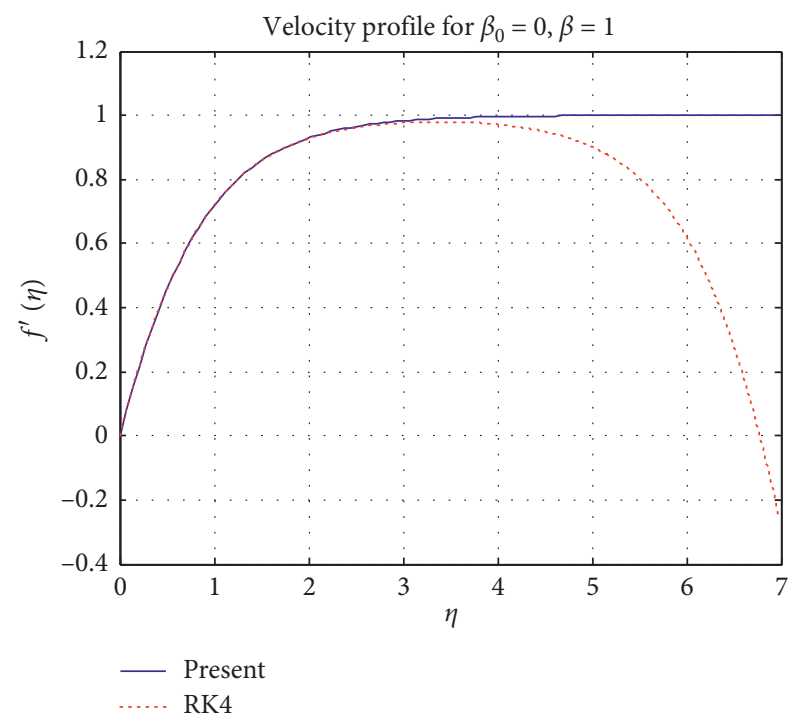

Figure 5: Velocity profile of the Pohlhausen flow $\left(\beta_{0}=0\right.$ and $\left.\beta=1\right) . f^{\prime \prime}(0)=1.154700538379$ for $L=6.98476$.

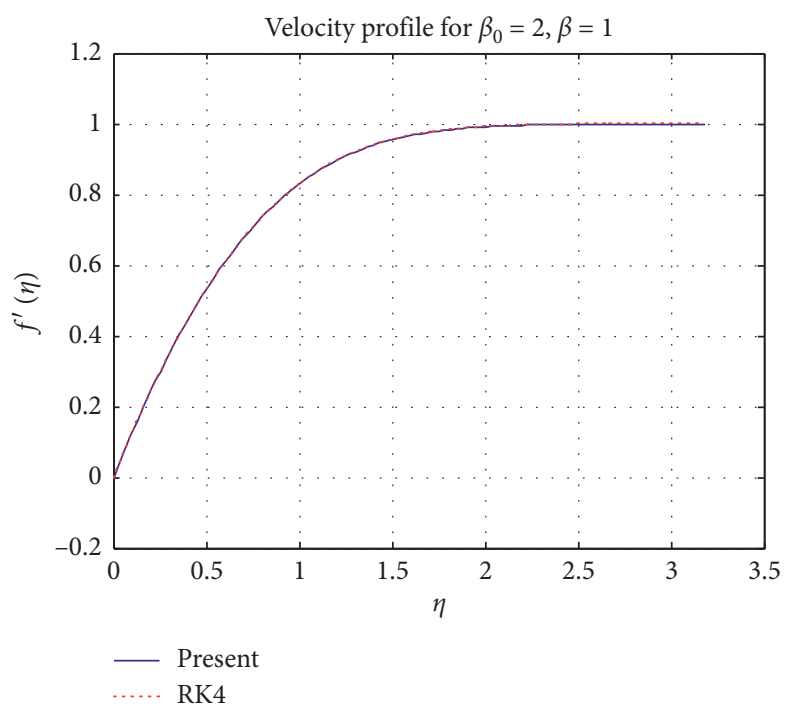

FIGURE 6: Velocity profile of the problem of Homann, describing steady flow in the boundary layer along a surface of revolution near the stagnation point $\left(\beta_{0}=2\right.$ and $\left.\beta=1\right) . f^{\prime \prime}(0)=1.311937693752$ for $L=3.18407676$.

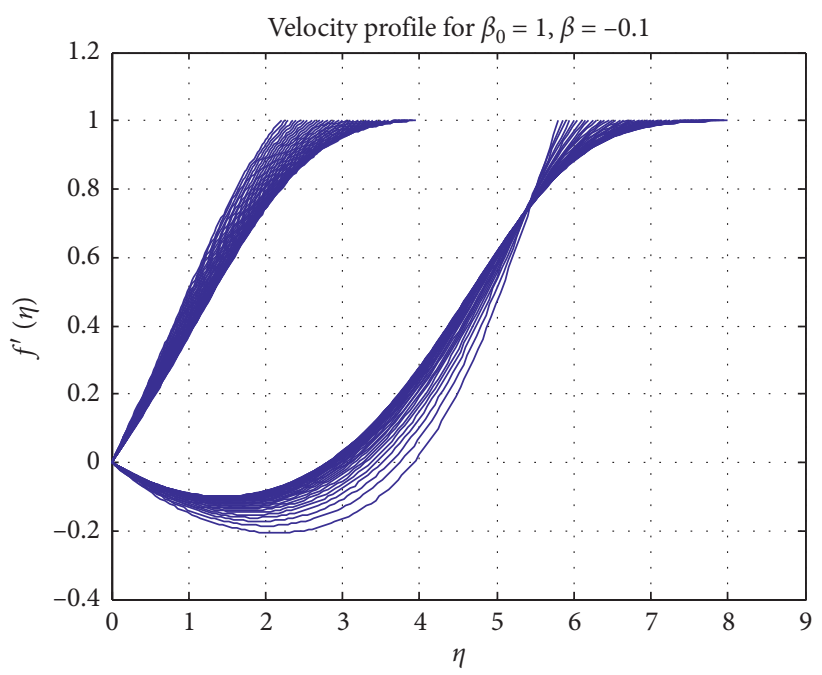

FigURE 7: Velocity profile for $\beta=-0.1$. 


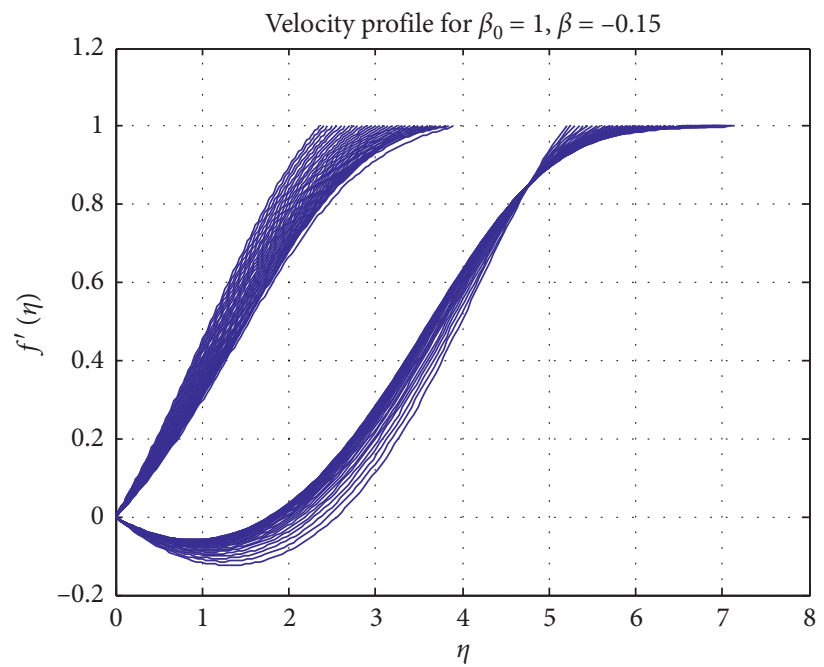

FIGURE 8: Velocity profile for $\beta=-0.15$.

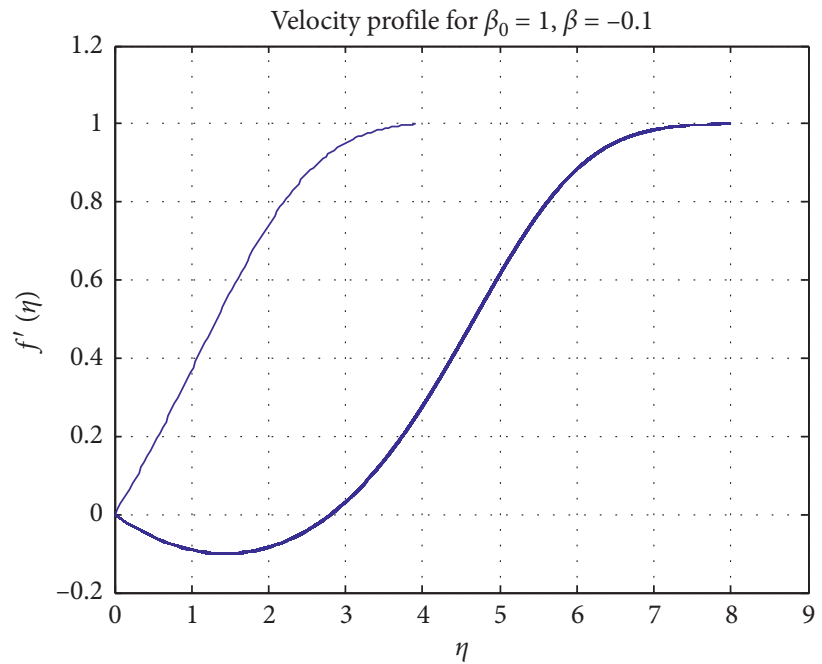

Figure 9: Velocity profile for $\beta=-0.1, \Delta L=0.001 ; f^{\prime \prime}(0)=0.326218125788279$ (Forward flow), $L=3.913 ; f^{\prime \prime}(0)=-0.140741363248159$ (reverse flow), $L=8$.

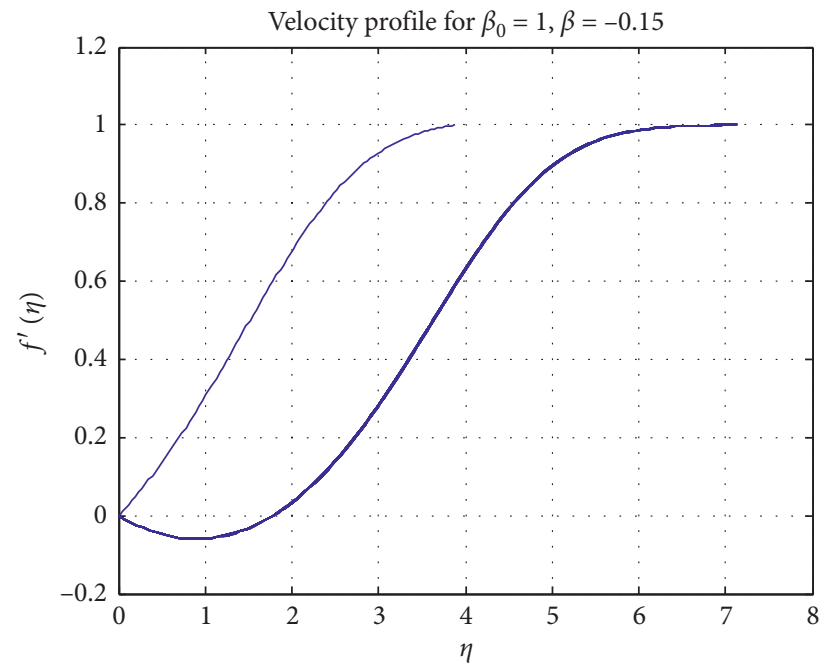

FiguRE 10: Velocity profile for $\beta=-0.15, \Delta L=0.001 ; f^{\prime \prime}(0)=0.233877086171824$ (Forward flow), $L=3.88 ; f^{\prime \prime}(0)=-0.133696366858524$ (reverse flow), $L=7.135$. 


\section{Conclusions}

In the present study, a technique based on the Chebyshev series expansion is applied to determine an approximate solution for the Falkner-Skan (F-S) third-order nonlinear differential equation. The nonlinear differential equation has been transformed into a system of algebraic equations which was presented in a matrix form. This system of equations is easily solved by matrix inversion, by assuming initial values for chebyshev coefficients and iterating this process until the correct values are within acceptable error. The new proposed technique is straightforward and well adapted to the computer implementation. One of the advantages of this method is that the solution is expressed as a system of algebraic equations which is directly solved using the computer program without any computational effort.

A comparison of the present results with the published results indicates excellent accuracy of the solution. It is concluded that the present technique is an accurate tool in handling the Falkner-Skan (F-S) equation with a high level of accuracy.

This technique can be considered as a powerful tool to solve the linear and nonlinear differential equations defined on a finite range.

\section{Data Availability}

All data and analysis are allowed to be shared by researchers throughout the published paper.

\section{Conflicts of Interest}

The authors declare that they have no conflicts of interest.

\section{References}

[1] M. Dehghan, "Numerical solution of a non-local boundary value problem with Neumanns boundary conditions," Communications in Numerical Methods in Engineering, vol. 19, no. 1, pp. 1-12, 2003.

[2] M. Dehghan, "Numerical procedures for a boundary value problem with a non-linear boundary condition," Applied Mathematics and Computation, vol. 147, no. 1, pp. 291-306, 2004.

[3] A. Saadatmandi, M. Razzaghi, and M. Dehghan, "SincGalerkin solution for nonlinear two-point boundary value problems with applications to chemical reactor theory," Mathematical and Computer Modelling, vol. 42, no. 11-12, pp. 1237-1244, 2005.

[4] A. Saadatmandi and M. Razzaghi, "The numerical solution of third-order boundary value problems using sinc-Collocation method," Communications in Numerical Methods in Engineering, vol. 23, no. 7, pp. 681-689, 2007.

[5] A. Saadatmandi and J. A. Farsangi, "Chebyshev finite difference method for a nonlinear system of second-order boundary value problems," Applied Mathematics and Computation, vol. 192, no. 2, pp. 586-591, 2007.

[6] A. Saadatmandi and M. Dehghan, "The numerical solution of a nonlinear system of second-order boundary value problems using sinc-collocation method," Mathematical and Computer Modelling, vol. 46, no. 11-12, pp. 1434-1441, 2007.
[7] M. Dehghan and M. Lakestani, "Numerical solution of nonlinear system of second-order boundary value problems using cubic B-spline scaling functions," International Journal of Computer Mathematics, vol. 85, no. 9, pp. 1455-1461, 2008.

[8] M. Lakestani, "Numerical solution for the Falkner-Skan equation using Chebyshev cardinal functions," Acta Universitatis Apulensis, vol. 27, pp. 229-238, 2011.

[9] K. Parand, N. Pakniat, and Z. Delafkar, "Numerical solution of the Falkner-Skan equation with stretching boundary by collocation method," International Journal of Nonlinear Science, vol. 11, no. 3, pp. 275-283, 2011.

[10] A. O. El-Nady and M. F. Abd Rabbo, "A new technique for solution of the Blasius and Falkner-Skan boundary layer equations," IOSR Journal of Mechanical and Civil Engineering (IOSRJMCE), vol. 14, no. 4, pp. 45-53, 2017.

[11] A. O El-Nady and M. F. Abd Rabbo, "Solution of FalknerSkan flow and heat transfer over a wedge by shooting method, Okasha," Journal of Scientific and Engineering Research, vol. 4, no. 12, pp. 413-423, 2017.

[12] T. Cebeci and H. B. Keller, "Shooting and parallel shooting methods for solving the Falkner-Skan boundary-layer equation," Journal of Computational Physics, vol. 7, no. 2, pp. 289-300, 1971.

[13] T. Y. Na, Computational Methods in Engineering Boundary Value Problems, Academic Press, New York, NY, USA, 1979.

[14] N. S. Asaithambi, "A numerical method for the solution of the Falkner-Skan equation," Applied Mathematics and Computation, vol. 81, no. 2-3, pp. 259-264, 1997.

[15] A. Asaithambi, "A finite-difference method for the FalknerSkan equation," Applied Mathematics and Computation, vol. 92, no. 2-3, pp. 135-141, 1998.

[16] N. S. Elgazery, "Numerical solution for the Falkner-Skan equation," Chaos, Solitons and Fractals, vol. 35, no. 4, pp. 738-746, 2008.

[17] B. D. Ganapol, "Highly accurate solutions of the Blasius and Falkner-Skan boundary layer equations via convergence acceleration," 2010, https://arxiv.org/abs/1006.3888.

[18] A. Akyüz-Daşcioğlu and H. Çerdi, “The solution of highorder nonlinear ordinary differential equations by Chebyshev series," Applied Mathematics and Computation, vol. 217, no. 12, pp. 5658-5666, 2011.

[19] Y. Öztürk and M. Gülsu, "The approximate solution of highorder nonlinear ordinary differential equations by improved collocation method with terms of shifted Chebyshev polynomials," International Journal of Applied and Computational Mathematics, vol. 2, no. 4, pp. 519-531, 2016.

[20] A. Okasha and H. M. Negm, "Matrix formulation of Chebyshev solution to shell problems," in Proceedings of the Tenth International Conference on Aerospace Science and Aviation Technology, Military Technical College, Cairo, Egypt, May 2003. 